\title{
The Potentialities of Coherent X-Radiation for Self-Assembled Systems and Liquid Crystal Research
}

\author{
V. B. Gavrikov ${ }^{1}$, V. P. Likhachev ${ }^{2}$, A. M. Figueiredo Neto ${ }^{2}$, J. D. T. Arruda-Neto ${ }^{2,3}$, \\ A. L. Bonini ${ }^{2}$, A. C. S. Lima ${ }^{2}$, S. Simionato ${ }^{2}$, and P. Iacomo Jr. ${ }^{1}$ \\ ${ }^{1}$ Kharkov Institute of Physics and Technology, 1 Akademishna 61108, Kharkov, Ukraine \\ ${ }^{2}$ Instituto de Física da Universidade de São Paulo, C.P. 66318, 05315-970, São Paulo, SP, Brazil \\ ${ }^{3}$ Universidade de Santo Amaro, Rua Prof. Enéas da Siqueira Neto 340, 04829-300, São Paulo, SP, Brazil
}

Received on 9 September, 2003

\begin{abstract}
The potentialities of coherent X-radiation for investigation of structural features of self-assembled systems and liquid crystals was studied using Monte Carlo techniques. The radiation spectra were simulated for the case of interaction of $20 \mathrm{MeV}$ electrons with a liquid crystal, and the influence of the various crystal parameters was studied. The simulations show that coherent X-radiation spectra are almost free of background, and that such measurements could be considered as an alternative to those where Synchrotron sources are used.
\end{abstract}

\section{Introduction}

Coherent X-radiation (CXR) is the coherent part of bremsstrahlung ([1]-[3]), which is produced by a relativistic particle traversing a crystalline target with the condition that the radiation energies are far from the atomic resonance energies. At the present time the properties of CXR, generated by electrons with energy of several tens of $\mathrm{MeV}$, have been throughout investigated for silicon and germanium [4]- [6], and diamond crystals [7]. At these electron energies features of the incoherent radiation component were studied also in [9] and [10].

The studies show advantages of the X-ray sources designed in terms of CXR since this radiation is quasimonochromatic, easy tunable, polarized and almost free of background. Nevertheless, the main drawback of such radiation is its relatively low intensity. For typical operation conditions, the CXR source intensity is few orders of magnitudes lower in comparison to a Synchrotron radiation source [8].

The low intensity of the CXR radiation for moderate electron beam energies makes problematic the design of $\mathrm{X}$ ray sources which could be competitive in intensity with a Synchrotron source.

To find a field of application for CXR, which uses its advantages and does not require high intensity, is a current and valid goal.

On the other hand, CXR characteristics, such as radiation intensity and orientation dependency on the energy of the coherent photons, depend strongly on structural features of the crystal sample. This fact allows to use CXR as an instrument to study the structure of biologically important macromolecular crystals, self-assembled systems, as well as phase transitions in such systems.

In this way, the principal disadvantage of CXR could be avoided since the same CXR process is used for photon production and characterization of the crystal sample. The interaction of real photons with a sample (X-ray source) is substituted by interaction of virtual photons. In a traditional scheme, a X-ray source of CXR consists of a two step process: first, a crystal is used for production of photons and, next, these photons are used for characterization of a sample.

In the present work we study the potentiality of CXR to study macromolecular crystal properties. In particular, the sensibility of CXR spectra to unit cell dimensions, distribution of electron density, phase transitions and crystal defects are all studied and addressed.

\section{Formalism}

The CXR is a result of two processes and its interference:

(i) bremsstrahlung emitted by a relativistic charged particle-projectile in the field of crystal atoms; and

(ii) the bremsstrahlung emitted by bound atomic electrons in the field of a projectile.

We start from the full amplitude of the radiation process for a non relativistic atom

$$
\begin{gathered}
\left|M_{a t}\right|^{2}=\left|M_{B S}+M_{P R}\right|^{2}= \\
\frac{2^{5} \pi^{3}}{\omega}\left|\overrightarrow{e_{f}}\left[\frac{e e_{0}^{2}}{m_{0} \gamma q^{2}}(Z-F(\vec{q})) \frac{\vec{q}}{\omega-\vec{k} \vec{v}}+\frac{e^{2} e_{0}}{m} \frac{F(\vec{q})}{\omega} \frac{\vec{v} \omega-\vec{q}}{(\vec{q}+\vec{k})^{2}-\omega^{2}}\right]\right|^{2},
\end{gathered}
$$


where $M_{B S}$ describes bremsstrahlung emitted by projectile; $M_{P R}$-bremsstrahlung emitted by bound atomic electrons; $\vec{v}, \gamma, e_{0}$ and $m_{0}$ are the projectile velocity; Lorentz factor, charge and mass, respectively; $\vec{k}, \overrightarrow{e_{f}}$ and $\omega$ are the momentum, vector of polarization and energy of the emitted photon, respectively; $\vec{q}$ is the recoil momentum, $Z$ is the atomic number and $F(\vec{q})$ is the atomic form factor.

In the case of a crystal, each atom contributes to the radiation amplitude with the factor:

$$
M_{a t} \exp \left(-i \vec{q} \overrightarrow{R_{\vec{L}}}\right),
$$

where $\overrightarrow{R_{\vec{L}}}$ is the relative coordinate of crystal atoms, and the cross section for a crystal differ from the atomic one by the diffraction factor:

$$
D(\vec{q})=\sum_{\overrightarrow{R_{\vec{L}}}} \exp \left(-i \vec{q} \overrightarrow{R_{\vec{L}}}\right) .
$$

In the case of a real solid crystal, the positions of the atoms fluctuate over their equilibrium positions as a result of thermal vibration and the diffraction factor should be averaged over these fluctuations by introducing the DebyeWaller factor $\exp \left(-\mathrm{q}^{2} \mathrm{u}^{2}\right)$, where $\mathrm{u}^{2}$ is the mean square temperature displacement of the crystal atoms from their equilibrium positions.

In the case of a liquid crystal [14], a periodic-type crystal structure exists only on limited distances and the correlation between atom positions practically disappears after some periods. The additional comparison with solid crystal degree of freedom results in fluctuation of lattice positions additionally to thermal fluctuation of atom positions. This liquid crystal property was introduced by random generation of lattice positions consecutively, with respect to each other, according to a Gauss distribution with a new mean square deviation $u_{0}$. We will consider here a lyotropic liquid crystal. Lyotropics [12] are mixtures of amphiphilic molecules and a solvent that, under proper temperature and concentration conditions, present self-assembled amphiphilic molecule aggregates. In the present case we will consider water as the solvent.

The connection between the energy $\omega$ of coherent photons and $\vec{k}, \vec{v}$ and $\vec{g}$ is defined by the $\delta$-function argument, and it is given by $\omega=g v \sin \varphi /\left(1-v \cos \theta_{\gamma}\right)$.

Calculations based on Eq. (1) implies the use of the freeatom approximation, where bounding effects between atoms within the molecules are ignored.

Eq. (1) presents the coherent component of the radiation in the coordinate system which is connected with an individual projectile moving through a target without scattering. Real radiation spectra, taking into account both multiple scattering process and photon attenuation inside the target, must be calculated in the laboratory coordinate system, where the $z$ axis is directed along the particle beam direction, and the $x z$-plane passes through the beam axis and the photon channel axis. For a target of thickness $L$ the number of photons per incident electron is

$$
N(\omega)=\int d \vec{v}_{\perp} f\left(\vec{v}_{\perp}, t\right) N_{0}(\omega) \exp \frac{-(L-t)}{L_{a}(\omega)} d t,
$$

where

$$
N_{0}(\omega)=N_{1}\left(d^{3} \sigma_{c o h}+d^{3} \sigma_{i n c}\right)+N_{2} d^{3} \sigma,
$$

The incoherent contributions to the radiation spectra arise from the compound system "water + amphiphilic aggregate". $d^{3} \sigma_{i n c}$ accounts for the incoherent contributions to the total radiation yield from the amphiphilic aggregate, and $d^{3} \sigma$ describes the incoherent radiation due to the water surroundings. These contributions are calculated accordingly to ref. [9] in the framework of a Monte-Carlo approach. For the water radiation contribution, the incoherent diffraction factor (see Eq.(1) in [9]) is equated to 1. In Eq. (4) $\overrightarrow{d v}_{\perp} f\left(\vec{v}_{\perp}, t\right)$ is the distribution of $\vec{v}_{\perp}$ along the depth of the target $t, N_{1}$ and $N_{2}$ are the number of the carbonic chains of the amphiphilic molecules and the water molecules per $\mathrm{cm}^{2}$ of the target, respectively. The integration in Eq. (4) is over the liquid crystal thickness and over the component of $\vec{v}$ perpendicular to the initial beam direction. The exponential factor takes into account the photon attenuation, and $L_{a}(\omega)$ is the absorption length. The calculations through Eq. (4) use the Monte Carlo simulation code described in [11].

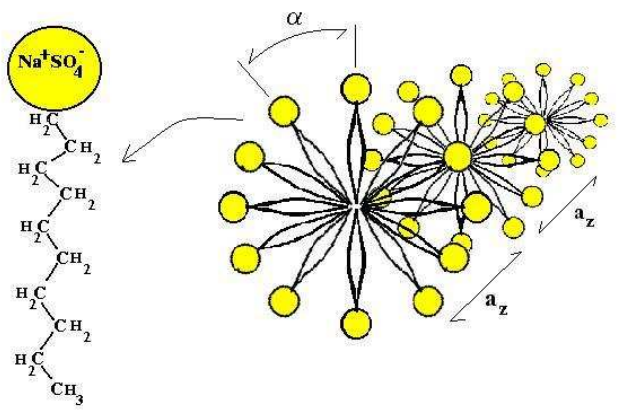

Figure 1. Amphiphilic molecule of the liquid crystal model used for simulation of CXR (see text for details).

To demonstrate the potentiality of CXR to study liquid crystal properties we use a simplified model for a lyotropic liquid crystal [12]. We consider that this liquid crystal is formed by amphiphilic molecules, sketched in Fig. 1, arranged in cylinders, and surrounded by water. According to this model, each amphiphilic aggregate has the form of a cylinder with radius $r$, and it can be built up by translating an "unit cell" at a distance $a_{z}$ along the cylindrical axis. As shown in Fig. 1, the unit cell is made up by placing along the radius the molecular complexes, which are constituted, for example, by molecules of Sodium decylsulfate $\left(\mathrm{NaSO}_{4} \mathrm{C}_{10} \mathrm{H}_{21}\right)$. The angle between the axes of the molecules is $\alpha$, and they can be oriented either "from" (I) 
or "to" (II) the center of the unit cell. In the plane, which is orthogonal to the molecular axes, the chains are arranged at equal distances $a_{x}$ and $a_{y}$ along the corresponding coordinate axes. The space between the cylindrical aggregates is uniformly filled with water.

\section{Results}

Using Monte Carlo technics [11] we simulate the CXR and incoherent radiation from the liquid crystal described above, to study the dependence of radiation characteristics versus crystal parameters.

Figure 2 shows the geometry for measuring CXR spectra and illustrates the definitions here employed. The $z$-axis is directed along the electron beam direction, $\vec{k}$ is the photon wave vector, $\theta_{\gamma}$ is the observation angle and $\varphi$ is the angle between a selected plane of the aggregate and the $z$-axis. When the axes of all aggregates lie on the vertical dashed lines, the crystal has a perfect structure. A departure from this structure is described by the mean-square deviation $u^{2}$.

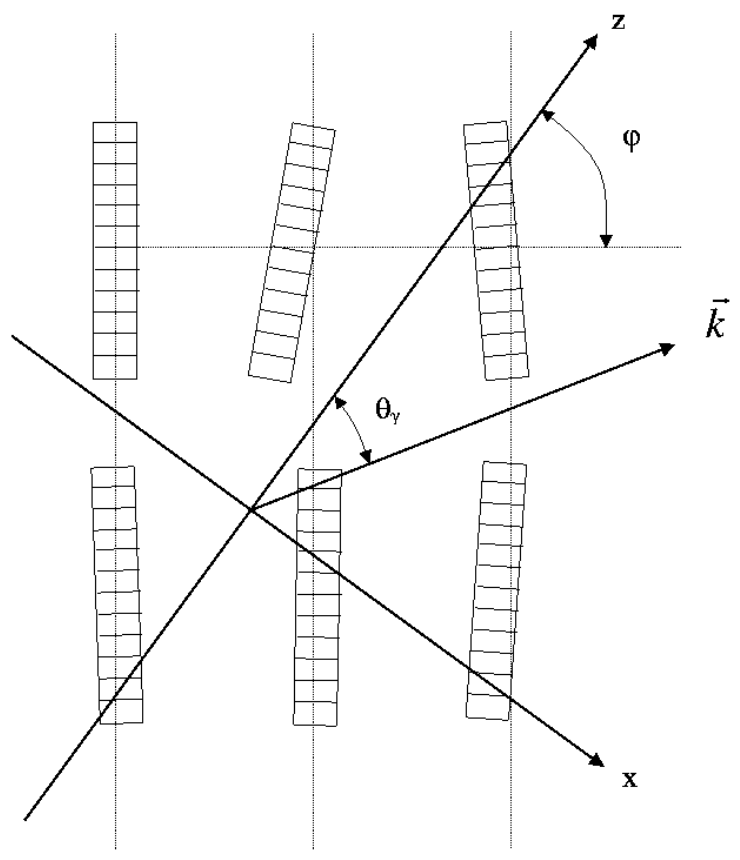

Figure 2. The CXR measuring geometry with the notation used in the text.

Figures $3 \mathrm{a}$ and $3 \mathrm{~b}$ show the radiation spectra resulting from the interaction of $20 \mathrm{MeV}$ electrons with the liquid crystal, which has $a_{z}=8 \AA, r=19 \AA$, and $\alpha=24^{\circ}$. The CXR intensity achieves its maximum values at $\varphi=$ $\theta_{\gamma} / 2 \pm 1 / 2 \gamma$ ( [5]) and for Figs. 3a and $3 \mathrm{~b}$ we took $\theta_{\gamma}=$ $45^{\circ}$ and $\varphi=21.8^{0}$. The energy resolution of the detector was considered to be equal to $10 \mathrm{eV}$ for the (100), and (110) planes and $20 \mathrm{eV}$ for the (001) plane. Such energy resolutions can be obtained by using tantalum-based superconducting tunnel junctions ([13]). All spectra were calculated for the case of I-orientation (Fig. 1). In the case of II-orientation the peaks are bigger by a factor 1.18 . This difference arises from the higher electron densities near the unit cell center for the case of II-orientation.
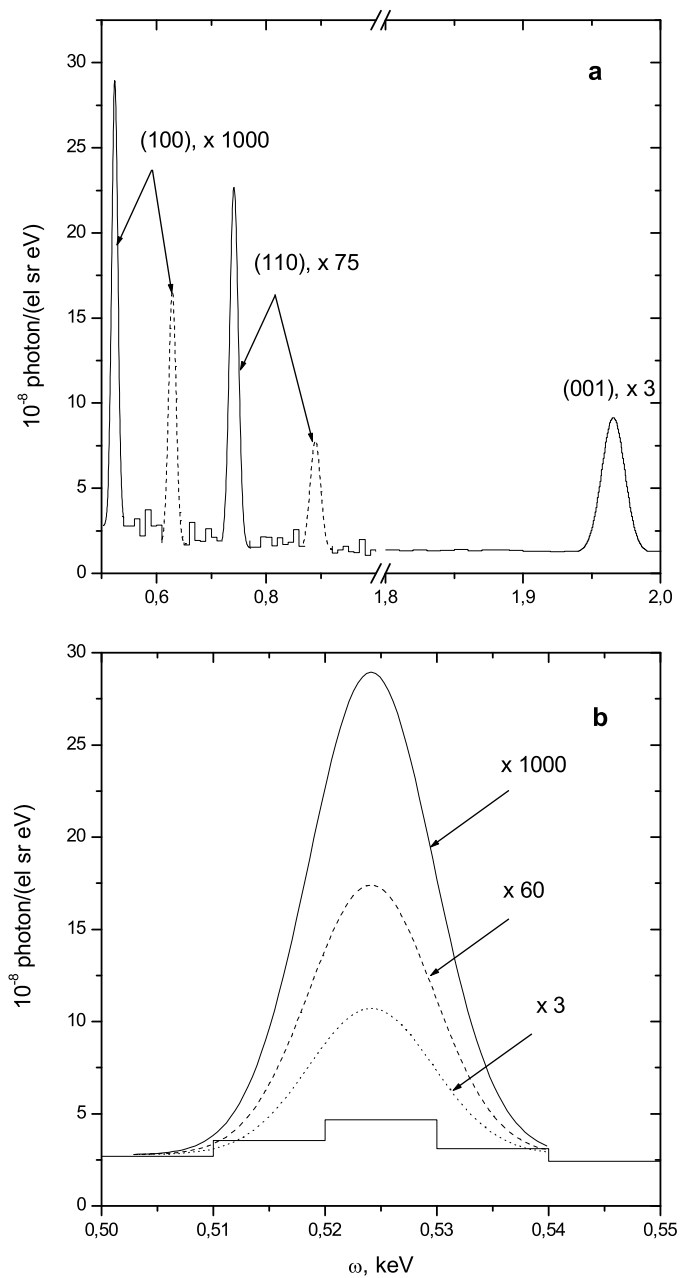

Figure 3. Radiation spectra, calculated for an electron beam with energy of $20 \mathrm{MeV}, \theta_{\gamma}=45^{\circ}, \varphi=21.8^{\circ}$, and for macromolecular crystal having $a_{z}=8 \AA, r=19 \AA, \alpha=24^{\circ}$, and for the case of Iorientation (see Fig. 1). a)-solid lines correspond to $a_{x}=a_{y}=30$ $\AA$, and for the dashed lines $-a_{x}=a_{y}=25 \AA$; for the (100) and (110) planes $u=5 \AA$, and for the (001) plane $u=2.5 \AA$. b)-plane (100) and various mean square deviations: $u=5 \AA$ (solid line), 10 $\AA$ (dashed line) and $13.5 \AA$ (dotted line).

In Fig. 3a the CXR spectrum is shown for the (100), (110) and (001) planes. For the (100) and (110) planes the mean square deviation was taken as $u=5 \AA$, and for the (001) plane as $u=2.5 \AA$. The solid lines correspond to the case $a_{x}=a_{y}=30 \AA$. The dashed peaks demonstrate how the coherent photon energies change by changing the inter-aggregate distances, and these peaks correspond to $a_{x}=a_{y}=25 \AA$.

Figure $3 b$ illustrates for the (100) plane how the effect of the radiation yield decreases when $u$ is increasing. The solid, dashed and dotted lines correspond to $u=5 \AA$, $10 \AA$, 
and $13.5 \AA$, respectively. The step line shows the contribution to the total radiation yield resulting from the incoherent radiation component.

\section{Conclusions}

Our results show that CXR experiments with liquid crystals can give a comprehensive information about structural features of the samples, and they can be considered as an alternative to those investigations where sources of photons, like the synchrotron, are used. At large observation angles CXR spectra are almost free of radiation background and, therefore, fine details of the sample structure can be studied. Our simulations show that for an electron beam current of $0.1 \mu A$ and a solid angle $d \Omega_{\gamma}=2 \cdot 10^{-5}$ sr, $3 \%$ of statistical error can be obtained for the (100) peak at exposition time around 1 second, and for the (001) peak at 1.5 minutes. Such time characteristics could be useful in studying phase transitions in liquid crystals.

\section{References}

[1] M.Ya. Amusia, Phys. Rep. 162, 5, 249 (1988).

[2] M.Ya. Amusia et al., Sov. Phys. JETP 61, 224 (1985).

[3] V.N. Tsytovich, I.M. Oiringel (eds.), Polarization Bremsstrahlung (Plenum Publ., New York - London, 1992).
[4] D.I. Adejishvili, V.B. Gavrikov, and V.A. Romanov, Nucl. Instrum. Meth. Phys. Res. B 152, 406 (1999).

[5] V.B. Gavrikov, V.P. Likhachev, M.N. Martins, and V.A. Romanov, Brazilian Journal of Physics, 29, 516 (1999).

[6] V.B. Gavrikov, V.P. Likhachev, and V.A. Romanov, Nucl. Instrum. Meth. Phys. Res. A 457, 411 (2001).

[7] J. Freudenberger, V.B. Gavrikov, M. Galemann, et al., Phys. Rev. Lett., 74, 2487 (1995).

[8] W.R. Dix et al, Nucl. Inst. Meth. Phys. Res. A 314, 307 (1992).

[9] V. B. Gavrikov, V. P. Likhachev, J. D. T. Arruda-Neto, and A. L. Bonini, Phys. Rev. A 65, 022903 (2002).

[10] V. B. Gavrikov, V. P. Likhachev, J. D. T. Arruda-Neto, and A. L. Bonini, European Phys. Journ. A 12, 4, 487 (2001).

[11] V. B. Gavrikov, V. P. Likhachev, J. D. T. Arruda-Neto, and A. L. Bonini, "Simulation of coherent X-ray radiation produced by relativistic charged particles in crystals", preprint IF-1559/2003, IF USP São Paulo, Brazil.

[12] G.H. Brown, Advances in liquid crystal, vol. 1, Academic Press, New York, 1975.

[13] see http://astro.estec.esa.nl/SA-general/Research/

[14] P.G. de Gennes and J.Prost, The physics of liquid crystals, clarendon Press, Oxford (1993). Stj/STJ_tantalum.html and references therein. 